\title{
SAPHO Syndrome: A Chameleon in Medical Diseases
}

\author{
Ling Twohig ${ }^{\mathrm{a}, \mathrm{c}}$, Henderson Lopez ${ }^{\mathrm{a}}$, Michelle $\mathrm{Ngo}^{\mathrm{b}}$
}

\begin{abstract}
SAPHO (synovitis, acne, pustulosis, hyperostosis and osteitis) syndrome is a unique condition. Its rarity and variable presentation consummate its place within an elite group of elusory diagnosis. As a result, the diagnosis of SAPHO syndrome is often delayed by many years and is associated with increased medical cost, human suffering and worsened outcome. We present an atypical presentation of the SAPHO syndrome exhibiting an insidious onset of left hip pain with proximal femoral diaphyseal sterile osteitis along with severe weight loss that remained undiagnosed for over 5 years. We searched PubMed using SAPHO syndrome, unique SAPHO, SAPHO case study, SAPHO, CMRO and diaphysis as keywords for our literature review. The purpose of this case report is to emphasize a need for vigilance, timely recognition and prompt treatment. We further recommend aggressive early therapy for swift symptom resolution and improved medical outcome.
\end{abstract}

Keywords: SAPHO syndrome; SAPHO; CMRO and diaphysis

\section{Introduction}

In 1961, Windam and his team were the first to observe and describe an association between musculoskeletal pain and skin lesions which later became known as the SAPHO (synovitis, acne, pustulosis, hyperostosis and osteitis) syndrome [1-3]. SAPHO is a rare condition with a prevalence of 1 in 10,000 , affecting predominantly adults between ages of 30 - 50 years old, though as young as 15 months has been observed [4]. In the original French SAPHO cases, the gender distribution was ap-

Manuscript accepted for publication October 22, 2015

aDepartment of Internal Medicine, Arrowhead Regional Medical Center, 400 N Pepper Ave., Colton, CA 92324, USA

b Department of Rheumatology, Loma Linda University Medical Center, 11234 Anderson St., Loma Linda, CA 92354, USA

${ }^{\mathrm{c} C}$ Corresponding Author: Ling Twohig, Department of Internal Medicine, Arrowhead Regional Medical Center, 400 N Pepper Ave., Colton, CA 92324, USA.Email: twohil@spu.edu

doi: http://dx.doi.org/10.14740/jmc2332w proximately equal; however, later studies deemed female preponderance to be the majority $[5,6]$. Geographically, European reports are among the most common, and some cases from Australia and Asia have been cited; however, studies are infrequent from North America and Latin Americas. African Americans tend to exhibit more severe clinical presentation with heterogeneous musculoskeletal and cutaneous manifestations [7]. Due to the heterogeneous nature in clinical manifestation, SAPHO is often an elusive diagnosis. Its clinical and imaging findings are often variable or may not be present at all; thus diagnosis is often delayed by $4.5-9.1$ years [8]. Even more concerning is the astounding negative impact of SAPHO on the patient's general health and quality of life; therefore, early diagnosis and timely treatment is of utmost importance [8].

We searched PubMed using SAPHO syndrome, unique SAPHO, SAPHO case study, SAPHO, CMRO and diaphysis as keywords and other relevant references were retrieved.

The purpose of our paper is to showcase an atypical presentation of SAPHO syndrome and emphasize the need for vigilance, timely recognition and prompt treatment. We present a 64-year-old lady who arrived to our emergency department suffering from a progressively escalated and unexplained left thigh pain over the past 5 years, with 65 pounds of unintentional weight loss as a result of untreated SAPHO syndrome.

\section{Case Report}

A 64-year-old lady with past medical history of hypertension presented to the emergency department complaining of severe pain in her left thigh. Her pains started in 2009 and became significantly worsened in the last 6 months. She also noted a 65 pound of unintentional weight loss during this time. The patient began to experience a psoriatic, pruritic, desquamating and tender palmoplantar pustular rash which started about 3 years ago, progressed and worsened over the past 2 months. The rash started in her palms (Fig. 1) and in the last year, the same rash appeared on her medial aspect of her feet (Fig. 2) as well. Recent X-rays (Fig. 3, 4) at a prior hospital suggested possible malignancy or infection in her left femur, thus she was transferred for higher level of care.

On admission, the patient had decreased strength 3 out of 5 of her left lower extremity with escalating pain. Rashes on the palms and soles were identified. Laboratory workup are 


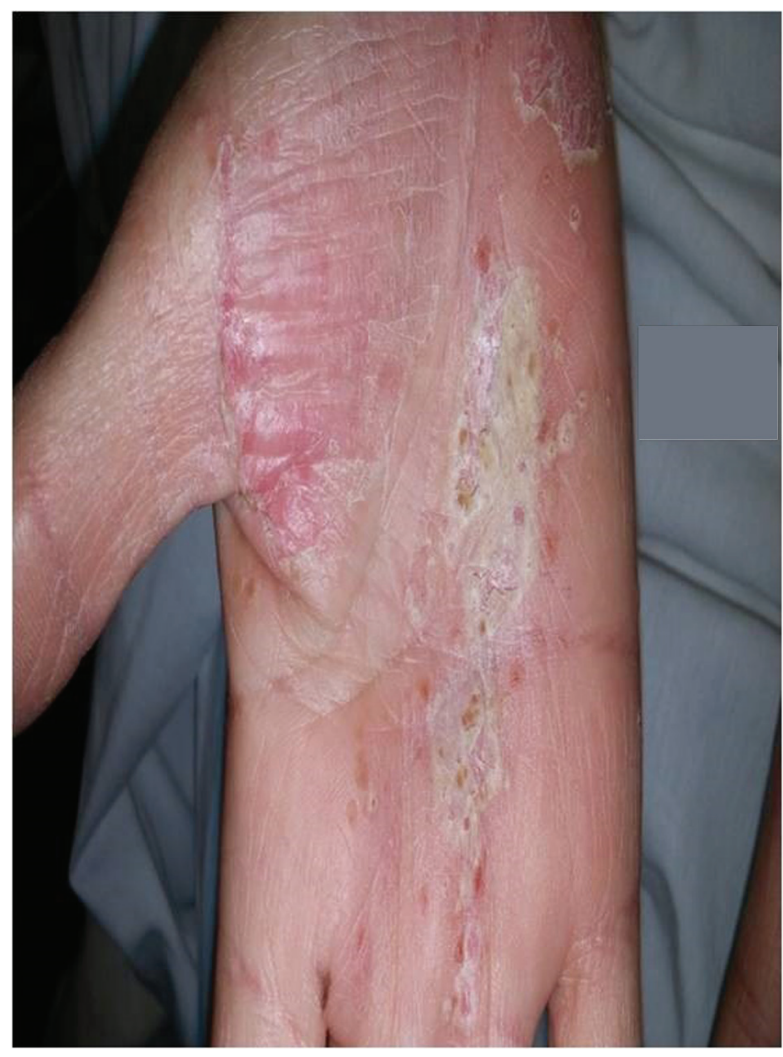

Figure 1. Skin biopsy of the right foot.

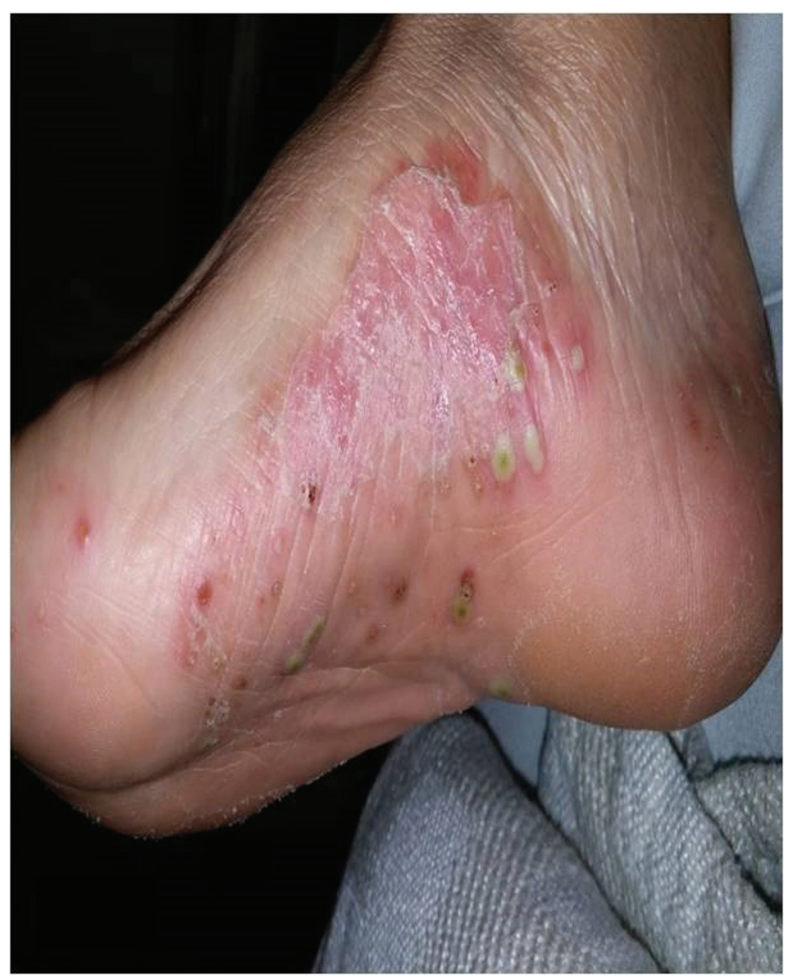

Figure 2. Right foot.

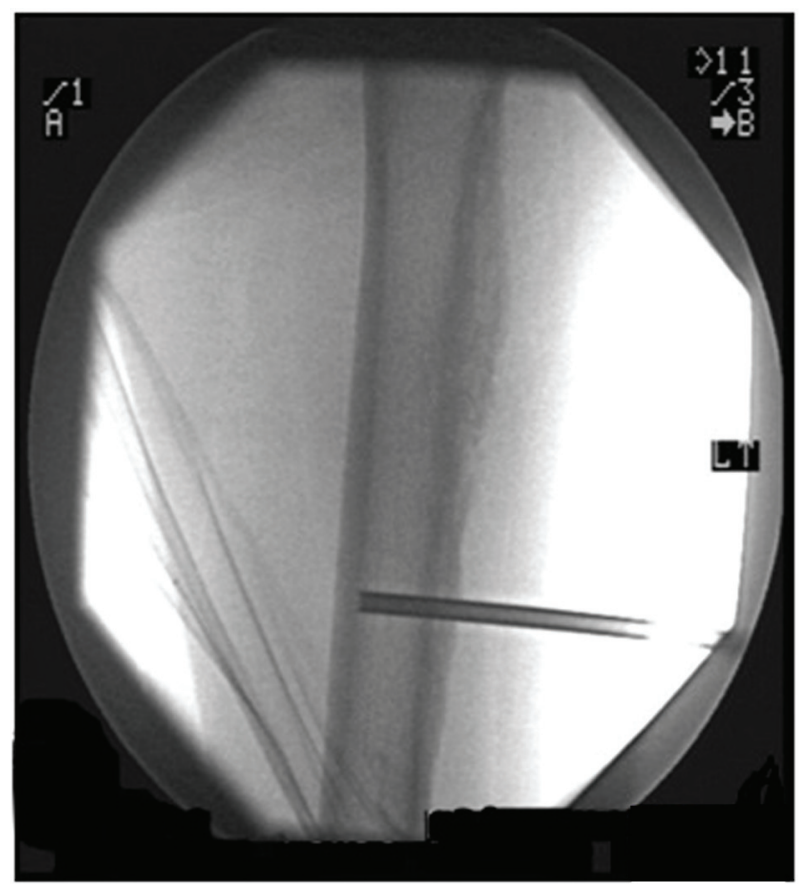

Figure 3. Skin biopsy of right palm.

negative for white blood cell (WBC) elevation, left shift in neutrophils, elevation in creatine kinase (CK-MB) or troponin. $\mathrm{XR}$ films demonstrate periosteal reaction of proximal diaphysis. The patient was placed on ketoconazole cream and oral steroid for the palmoplantar rashes.

On day two of hospitalization, a chest CT with contrast negated any signs of metastatic disease or primary neoplasm. Abdominal CT showed questionable thickened areas in sigmoid colon, left renal cyst, cystic lesions in right kidney, and

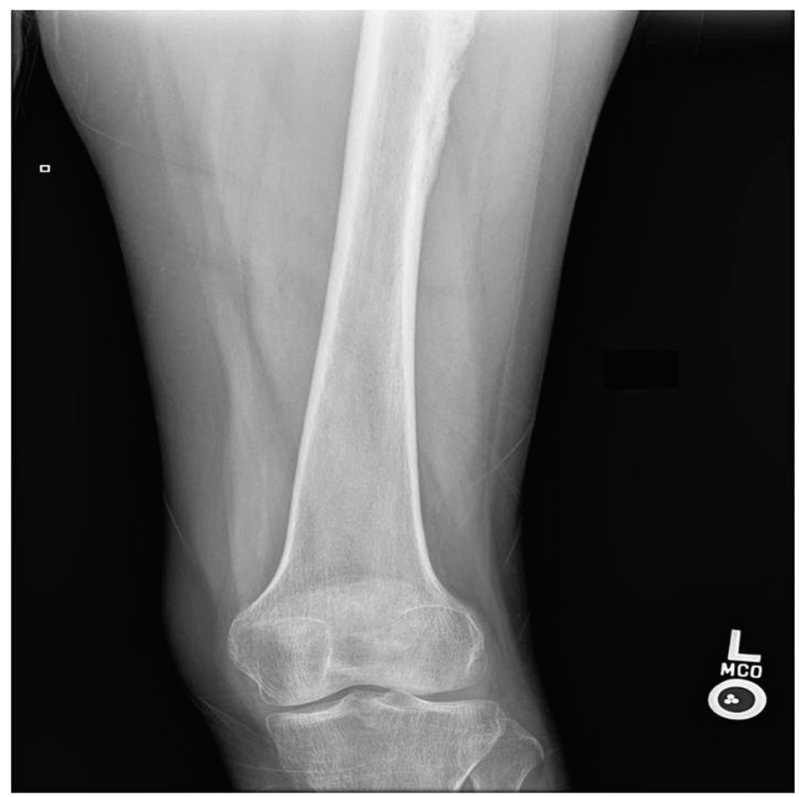

Figure 4. Right palm skin lesion. 


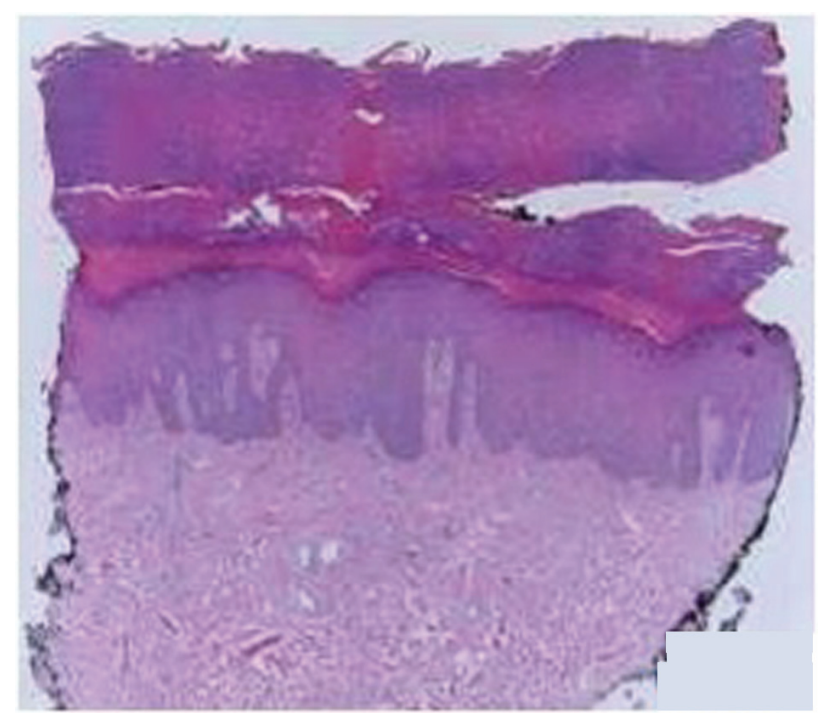

Figure 5. Femur under fluoroscopy.

sclerosis of the right sacral ala.

MRI of the left lower extremity demonstrated increased $\mathrm{T} 2$ and decreased T1 signals of the proximal diaphysis, and increased signals seen around the quadriceps muscle group, which are consistent with osteomyelitis and myositis, and less likely to be malignancy. Her whole body bone scan (technetium99m hydroxymethylene diphosphonate bone scan ${ }^{99 \mathrm{~m}} \mathrm{Tc} \mathrm{HDP}$ ) revealed abnormal activity in left proximal and mid femoral diaphysis, sternoclavicular joints, knees, shoulders, and ankles which favor an infection, and were unlikely to be malignant.

On day three, orthopedics and rheumatology are consulted to biopsy the left femur and dermatology for biopsy of the skin lesion.

Bone biopsy was taken by orthopedics with results showing reactive bony sclerosis, reactive fibrosis of the marrow space, no inflammation, no osteomyelitis nor evidence of tumors. Microscopically, mixtures of lamellar and reactive woven bone were seen. In addition, myxoid areas and hemosiderin were noted in the bone marrow space while the skin biopsy reveals sterile areas of palmoplantar pustulosis with hypertrophy of skin (Fig. 5, 6).

During the patient's stay, she was started on Norco $5 \mathrm{mg}$ PO Q6H, naproxen $500 \mathrm{mg}$ PO BID WC, amlodipine $5 \mathrm{mg}$ PO tablet daily, gabapentin $300 \mathrm{mg}$ PO capsule TID, ketorolac 15 mg IM once, ibuprofen $200 \mathrm{mg}$ PO tablet Q6H, ketoconazole cream and omeprazole $40 \mathrm{mg}$ PO capsule QAMAC. Ketoconazole cream showed no significant improvement in patient's rashes and was discontinued on day three. Upon discharge, the patient was given a course of naproxen for pain control and follow-up with rheumatology clinic.

After 1 month, the patient had continued bone pain which she was started on fosamax $75 \mathrm{mg}$ PO weekly, naproxen was switched to ibuprofen $800 \mathrm{mg}$ PO TID, and continuing physical therapy. For her persistent palmoplantar skin lesions, she was started on methotrexate $7.5 \mathrm{mg}$ PO once, and increased to $15 \mathrm{mg}$ PO weekly, along with folic acid of $1 \mathrm{mg}$ daily.

On her fourth month follow-up, her erythematous plaques

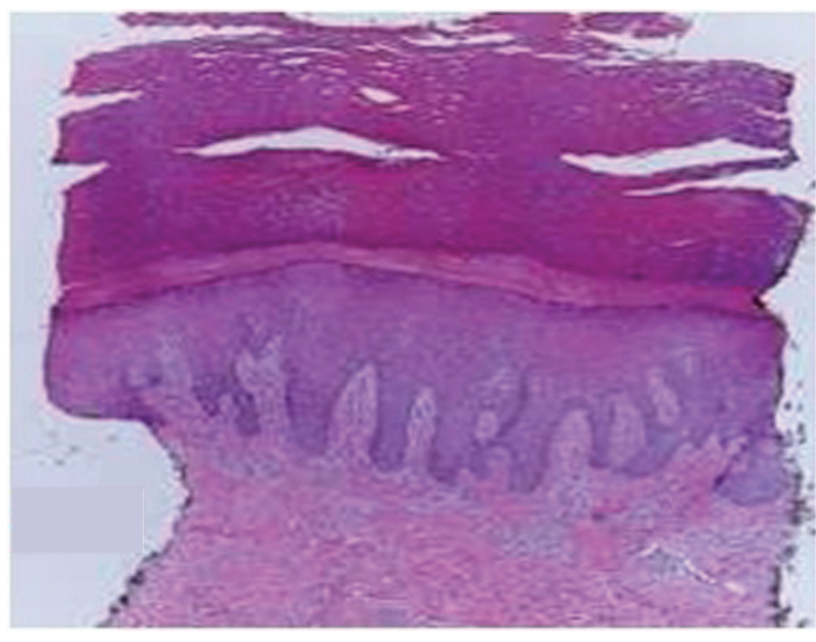

Figure 6. Femur under X-ray.

on the palms and soles were resolving, and she had no acneiform lesions. She was given clobetasol ointment for her dermatitis, and continued methotrexate and folic acid treatments.

\section{Discussion}

Diagnosis of SAPHO syndrome is challenging primarily due to its insidious onset of symptoms and often delayed presentation or absence of any specific host manifestation at the time of the evaluation.

SAPHO most commonly affects anterior chest wall with associated chest pain as a manifestation of enthesopathy, synovitis, osteitis or a combination of [7]. According to Nguyen et al, over $70 \%$ SAPHO syndrome would involve sternoclavicular manifestations, in comparison to appendicular skeleton involvement consisting of only $5-10 \%$ of cases. In addition, peripheral long bone involvement usually presents as osteolysis in the metaphysic adjacent to the growth plate with gradual transform to sclerosis with hyperostosis. Invariably, all patients will succumb to incapacitating pain frequently exacerbated by movement or pressure [5]. In contrast, our patient did not exhibit the classic chest/back or shoulder pain. Her osteoarticular symptoms consisted mainly of an unrelenting left thigh pain, with abnormal bony activities in multiple appendicular skeletal locations confirmed by ${ }^{99 \mathrm{~m}} \mathrm{Tc}$ HDP bone scan. Further, typical appendicular long bone involvements primarily affect the metaphysis adjacent to the growth plate [5]. Our patient demonstrated diaphyseal involvement. Her bone biopsy demonstrates reactive bony sclerosis with fibrosis that conforms to chronic bony changes as documented in prior case studies [5]. After infectious and malignant causes were ruled out, the suspicion of SAPHO syndrome grows stronger [9].

Another SAPHO's diagnostic criterion is the dermatologic manifestations that frequently accompany arthritic changes. The skin changes often are seen as severe acne and pustulosis palmoplantaris (PPP). Severe acne, specifically acne conglobata and acne fulminans, present in about $25 \%$ of patients [6]. PPP are detected in $50-75 \%$ of the SAPHO patients [10]. The 
musculoskeletal symptoms can occur before skin involvement in $40-68 \%, 30 \%$ simultaneously and late manifestation in 32 $60 \%$ of patients [7]. Our patient experienced the classic PPP, however, only in the later stages of her disease. Her pustular rash was 3 years after the onset of her leg pain, as comparison to other SAPHO cases where approximately $70 \%$ patients will exhibit skin manifestations within 2 years from the time of onset [4].

Because of the rarity of SAPHO syndrome, no randomized controlled trials have been conducted on the effectiveness of medical therapy. Therefore, treatments are based on published case reports and small case series with expert opinion. NSAIDs and analgesics are first line agents, though therapeutic effects are limited and approximately $50 \%$ of SAPHO patient would continue to suffer from pain and disease exacerbation [11, 12]. Alternatively, disease-modifying anti-rheumatic drugs (DMARDs) have had some success in some while ineffective in others. Bisphosphonates, known for its anti-bone resorption and anti-inflammation properties, are ideal for refractory SAPHO cases, often yielding considerable pain relief and sustained remission. As seen in our patient, who was initially treated and was refractory to NSAIDs therapy, her decade of unrelenting pain improved only after the initiation of methotrexate and fosamax [13].

Unrecognized SAPHO syndrome has a significant disease burden according to a German nationwide patient survey [8]. On a visual analogue scale from 0 to 100 , pains is logged at $45.4 \pm 25.9$, and mean impairment to daily activities is 62.3 \pm 27 . Patient questionnaire further demonstrates pronounced restriction in general health, social functioning, physical capacity, vitality, emotional and mental health [8]. Other possible complications (i.e. thrombosis, depressed mood) have been observed in SAPHO syndrome, further supporting the merits in early diagnosis and aggressive treatment $[13,14]$. As it was seen in our patient, after suffering 5 years of progressive pain which may be responsible for her eventual 65 lbs weight loss, early recognition and timely therapy could have reduced her suffering and general health deterioration.

\section{Conclusion}

SAPHO syndrome is difficult to diagnose due to its variable presentations and unknown etiologies. Its rarity prohibits large scale clinical trials to further illuminate this elusive syndrome; hence diagnoses are often delayed by many years. However, its high disease burden mandates physicians to remain vigilant with a high degree of clinical suspicion. The purpose of this case study is to raise awareness to a set of cognizant clinical features of SAPHO syndrome as well as its atypical manifestations.

\section{Acknowledgement}

The authors thank Dr. Torralba for a helpful discussion at the conception of this research.

\section{References}

1. Khanna L, El-Khoury GY. SAPHO syndrome--a pictorial assay. Iowa Orthop J. 2012;32:189-195.

2. Windom RE, Sanford JP, Ziff M. Acne conglobata and arthritis. Arthritis Rheum. 1961;4:632-635.

3. Chamot AM, Benhamou CL, Kahn MF, Beraneck L, Kaplan G, Prost A. [Acne-pustulosis-hyperostosis-osteitis syndrome. Results of a national survey. 85 cases]. Rev Rhum Mal Osteoartic. 1987;54(3):187-196.

4. Rukavina I. SAPHO syndrome: a review. J Child Orthop. 2015;9(1):19-27.

5. Nguyen MT, Borchers A, Selmi C, Naguwa SM, Cheema G, Gershwin ME. The SAPHO syndrome. Semin Arthritis Rheum. 2012;42(3):254-265.

6. Mari A, Morla A, Melero M, Schiavone R, Rodriguez J. Diffuse sclerosing osteomyelitis (DSO) of the mandible in SAPHO syndrome: a novel approach with anti-TNF therapy. Systematic review. J Craniomaxillofac Surg. 2014;42(8):1990-1996.

7. Carneiro S, Sampaio-Barros PD. SAPHO syndrome. Rheum Dis Clin North Am. 2013;39(2):401-418.

8. Witt M, Meier J, Hammitzsch A, Proft F, Schulze-Koops H, Grunke M. Disease burden, disease manifestations and current treatment regimen of the SAPHO syndrome in Germany: results from a nationwide patient survey. Semin Arthritis Rheum. 2014;43(6):745-750.

9. Kahn MF, Hayem F, Hayem G, Grossin M. Is diffuse sclerosing osteomyelitis of the mandible part of the synovitis, acne, pustulosis, hyperostosis, osteitis (SAPHO) syndrome? Analysis of seven cases. Oral Surg Oral Med Oral Pathol. 1994;78(5):594-598.

10. Zuo RC, Schwartz DM, Lee CC, Anadkat MJ, Cowen EW, Naik HB. Palmoplantar pustules and osteoarticular pain in a 42-year-old woman. J Am Acad Dermatol. 2015;72(3):550-553.

11. Hatano H, Shigeishi H, Higashikawa K, Shimasue H, Nishi H, Oiwa H, Shindo H, et al. A case of SAPHO syndrome with diffuse sclerosing osteomyelitis of the mandible treated successfully with prednisolone and bisphosphonate. J Oral Maxillofac Surg. 2012;70(3):626631.

12. Takeuchi K, Matsusita M, Takagishi K. A case of SAPHO (synovitis-acne-pustulosis-hyperostosis-osteomyelitis) syndrome in which $[18 \mathrm{~F}]$ fluorodeoxyglucose positron emission tomography was useful for differentiating from multiple metastatic bone tumors. Mod Rheumatol. 2007;17(1):67-71.

13. Freira S, Fonseca H, Ferreira PD, Vasconcelos P, Fonseca JE. SAPHO syndrome in an adolescent: a clinical case with unusual severe systemic impact. J Adolesc Health. 2014;55(2):304-306.

14. Carranco-Medina TE, Hidalgo-Calleja C, Calero-Paniagua I, Sanchez-Gonzalez MD, Quesada-Moreno A, Usategui-Martin R, Perez-Garrido L, et al. Thrombotic manifestations in SAPHO syndrome. Review of the literature. Reumatol Clin. 2015;11(2):108-111. 